\title{
Resolving the extended stellar halos of nearby galaxies: the wide-field PISCeS survey $\dagger$
}

\author{
D. Crnojevic ${ }^{1}$, D. J. Sand ${ }^{1}$, N. Caldwell ${ }^{2}$, P. Guhathakurta ${ }^{3}$, B.

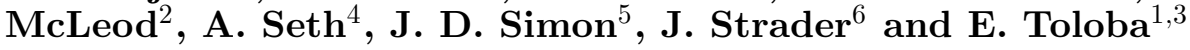 \\ ${ }^{1}$ Texas Tech University, Physics Department, Box 41051, Lubbock, TX 79409-1051, USA \\ email: denija.crnojevic@ttu.edu \\ ${ }^{2}$ Harvard-Smithsonian Center for Astrophysics, Cambridge, MA 02138, USA \\ ${ }^{3}$ UCO/Lick Observatory, University of California, Santa Cruz, 1156 High Street, Santa Cruz, \\ CA 95064, USA \\ ${ }^{4}$ Department of Physics and Astronomy, University of Utah, Salt Lake City, UT 84112, USA \\ ${ }^{5}$ Observatories of the Carnegie Institution for Science, 813 Santa Barbara Street, Pasadena, \\ CA 91101, USA \\ ${ }^{6}$ Michigan State University, Department of Physics and Astronomy, East Lansing, MI 48824, \\ USA
}

\begin{abstract}
In the wide-field Panoramic Imaging Survey of Centaurus and Sculptor (PISCeS), we investigate the resolved stellar halos of two nearby galaxies (the elliptical Centaurus A and the spiral Sculptor, D $\sim 3.7 \mathrm{Mpc}$ ) out to a projected galactocentric radius of $150 \mathrm{kpc}$ with Magellan/Megacam. The survey has led to the discovery of $\sim 20$ faint satellites to date, plus prominent streams and substructures in two environments that are substantially different from the Local Group, i.e. the Centaurus A group dominated by an elliptical and the loose Sculptor group of galaxies. These discoveries clearly attest to the importance of past and ongoing accretion processes in shaping the halos of these nearby galaxies, and provide the first census of their satellite systems down to an unprecedented $M_{V}<-8$. The detailed characterization of the stellar content, shape and gradients in the extended halos of Sculptor, Centaurus A, and their dwarf satellites provides key constraints on theoretical models of galaxy formation and evolution.
\end{abstract}

Keywords. galaxies: groups: individual (CenA, NGC253) — galaxies: halos — galaxies: dwarf — galaxies: photometry - galaxies: evolution - galaxies: luminosity function - galaxies: stellar content - galaxies: interaction

\section{Introduction}

The past decade has witnessed the advent of wide-field instrumentation, which has quickly led to the discovery of a wealth of stellar streams and faint satellites in our own Galaxy's halo (Ibata et al. 2001, Belokurov et al. 2006). Our nearest massive neighbor, M31, has been the next to be systematically surveyed out to large galactocentric radii, uncovering a similarly rich amount of substructures and satellites (McConnachie et al. 2009). The widely accepted $\Lambda$ CDM model for hierarchical structure assembly does indeed predict that the remnants of past/ongoing accretion and interaction events should populate the outskirts of galaxy halos, testifying their evolutionary history. Simulations also show a significant halo-to-halo scatter in the properties of galaxy halos, due to a

$\dagger$ This paper includes data gathered with the 6.5 meter Magellan Telescopes located at Las Campanas Observatory, Chile. 
wide variety in their assembly histories. However, the physical processes that regulate star formation and galaxy evolution (e.g. supernova feedback, reionization, environmental effects, etc.) remain poorly understood. While the detection of substructures in the outer halos of virtually all galaxies observed in great depth (Tal et al. 2009, Martinez-Delgado et al. 2010, Atkinson et al. 2013, Duc et al. 2015) agree well with theoretical predictions, to date they can only qualitatively confirm this picture: the intrinsic faintness of these features $\left(\mu_{V} \gtrsim 28 \mathrm{mag} / \operatorname{arcsec}^{2}\right)$ poses a challange to their detailed characterization beyond the Local Group (LG). To make things worse, there are obvious discrepancies between the predicted number and baryonic content properties of the smallest galaxies in our own LG (the "missing satellite" and "too big to fail" problems, e.g., Moore et al. 1999, Boylan-Kolchin et al. 2012). The faint end of the satellite luminosity function has recently started to be explored beyond the LG (e.g., M81, Chiboucas et al. 2013; M101, Merritt et al. 2014), and yet far fewer galaxies with $M_{V} \gtrsim-12$ are observed than predicted by simulations.

In order to test and put quantitative constraints on theoretical predictions, it is imperative to observe and characterize a larger sample of galaxies, with a range of morphologies and living in different environments: this has been the motivation for our Panoramic Imaging Survey of Centaurus and Sculptor (PISCeS), which we introduce in the next section.

\section{The PISCeS survey}

The PISCeS survey targets two nearby galaxies $(D \sim 3.7 \mathrm{Mpc})$ : Centaurus A (Cen A, or NGC 5128), the closest elliptical to us and the dominant galaxy of a dense group of galaxies, and Sculptor (NGC 253), a spiral located in a much looser and elongated group. Sculptor has a mass comparable to our own Milky Way (MW; Karachentsev et al. 2005), while Cen A is slightly more massive (Woodley et al. 2007), and due to their proximity they can be resolved into individual stars, thus allowing for a detailed comparison with the extant surveys of the MW, M31 and M81.

We use the optical Megacam imager at the 6.5-m Magellan II Clay telescope (McLeod et al. 2015): with a field-of-view of $24 \times 24 \operatorname{arcmin}^{2}$ and a binned pixel scale of $0.16 "$, this is an ideal instrument to deliver a deep, wide-field survey of our target galaxies. With the final goal of reaching projected galactocentric radii of $\sim 150 \mathrm{kpc}$ ( or $\sim 16 \mathrm{deg}^{2}$ ) in the halos of Cen A and Sculptor, since 2010 we have covered $\sim 13 \mathrm{deg}^{2}$ for each of them in the $g$ and $r$ filters (with $6 \times 300 \mathrm{~s}$ exposures). The survey is expected to be completed in 2016.

The standard image reduction and processing is performed by the Smithsonian Astrophysical Observatory Telescope Data Center, and subsequently resolved point spread function photometry is obtained from the stacked images with the DAOPHOT and ALLFRAME packages (Stetson 1987, 1994). Standard stars from SDSS are observed during clear nights to calibrate the data, and adjacent pointings are designed to overlap at the edges to ensure a good calibration under non photometric conditions as well. Finally, we perform artificial star tests in order to assess photometric uncertainties and incompleteness for each individual pointing (this is crucial for all such surveys given the non-uniformity in observing conditions from pointing to pointing).

The deep, resolved Magellan/Megacam images allow us to obtain color-magnitude diagrams (CMDs) such as the one shown in Fig. 1 (for Cen A's survey to date). Our survey reaches limiting magnitudes of $\sim 26.5-27 \mathrm{mag}$ in $r$-band, which corresponds to $\sim 1.5-2$ mag below the tip of the red giant branch (TRGB). A red giant branch (RGB) population at the distance of the target galaxy is clearly identified, as underlined by the 


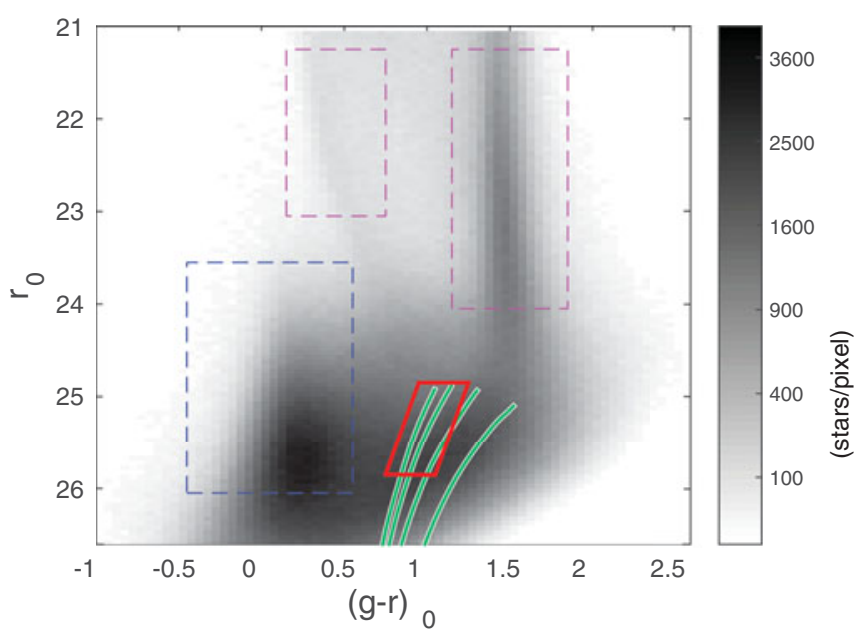

Figure 1. Dereddened Hess color-magnitude diagram $(0.05 \times 0.05$ mag bins $)$ for the Cen A PISCeS stellar catalogue to date. Dartmouth isochrones (Dotter et al. 2008) of 12 Gyr with a range of metallicities $([\mathrm{Fe} / \mathrm{H}]=-2.5$ to -1.0 in 0.5 dex steps) are shifted to Cen A's distance and overlaid. The RGB selection box is shown in red, while the contaminants' sequences are approximately delineated by dashed boxes (blue for unresolved galaxies at $(g-r)_{0} \sim 0.1$, magenta for MW halo and disk stars, at $(g-r)_{0} \sim 0.5$ and $(g-r)_{0} \sim 1.5$, respectively).

old isochrones with a range of metallicities, and is clearly separated from the contaminants' sequences (see Fig. 1).

\section{Preliminary results}

To illustrate the potential of our survey, we present the metal-poor RGB stellar density map of Cen A in Fig. 2, which includes the PISCeS pointings obtained to date (see Fig. 1 for the RGB selection box). The central regions of Cen A clearly show a variety of resolved features including shells and streams, which highlight the high degree of interaction recently experienced by this elliptical.

\subsection{Newly discovered satellites}

We use both visual inspection and the detection of overdensities in the resolved RGB stellar density maps in order to look for previously unknown faint satellites of Cen A and Sculptor. The discoveries of the first robust candidate satellites for both hosts are reported in Crnojević et al. (2014) and Sand et al. (2014). In total, to date we have identified 13 candidate Cen A satellites and 4 candidates for Sculptor, down to absolute magnitudes of $M_{V} \sim-8.0$. Resolved populations are clearly significantly more powerful than integrated light alone, given the otherwise prohibitively low surface brightness we are able to reach (down to $\mu_{V} \sim 32 \mathrm{mag} / \operatorname{arcsec}^{2}$ ). Nevertheless, some of our candidate satellites appear as surface brightness enhancements coupled with only a few resolved stars, and they may represent satellites even fainter than our detection limit. The newly discovered satellites resemble the properties (half-light radius, central surface brightness) of LG dwarfs with comparable luminosities (Crnojević et al. 2014, Sand et al. 2014, Crnojević et al. 2016, Toloba et al. 2016a).

Previously known Cen A satellites are easily recognizable in the stellar density map (blue circles in the lower panel of Fig. 2): ESO324-24 at $(\xi \sim 0.3, \eta \sim 1.6)$, NGC5237 at $(2.2,0.1)$, KKs55 at $(-0.7,0.3)$, KK197 at $(-0.75,0.5)$, as well as KK203 and KK196 in 

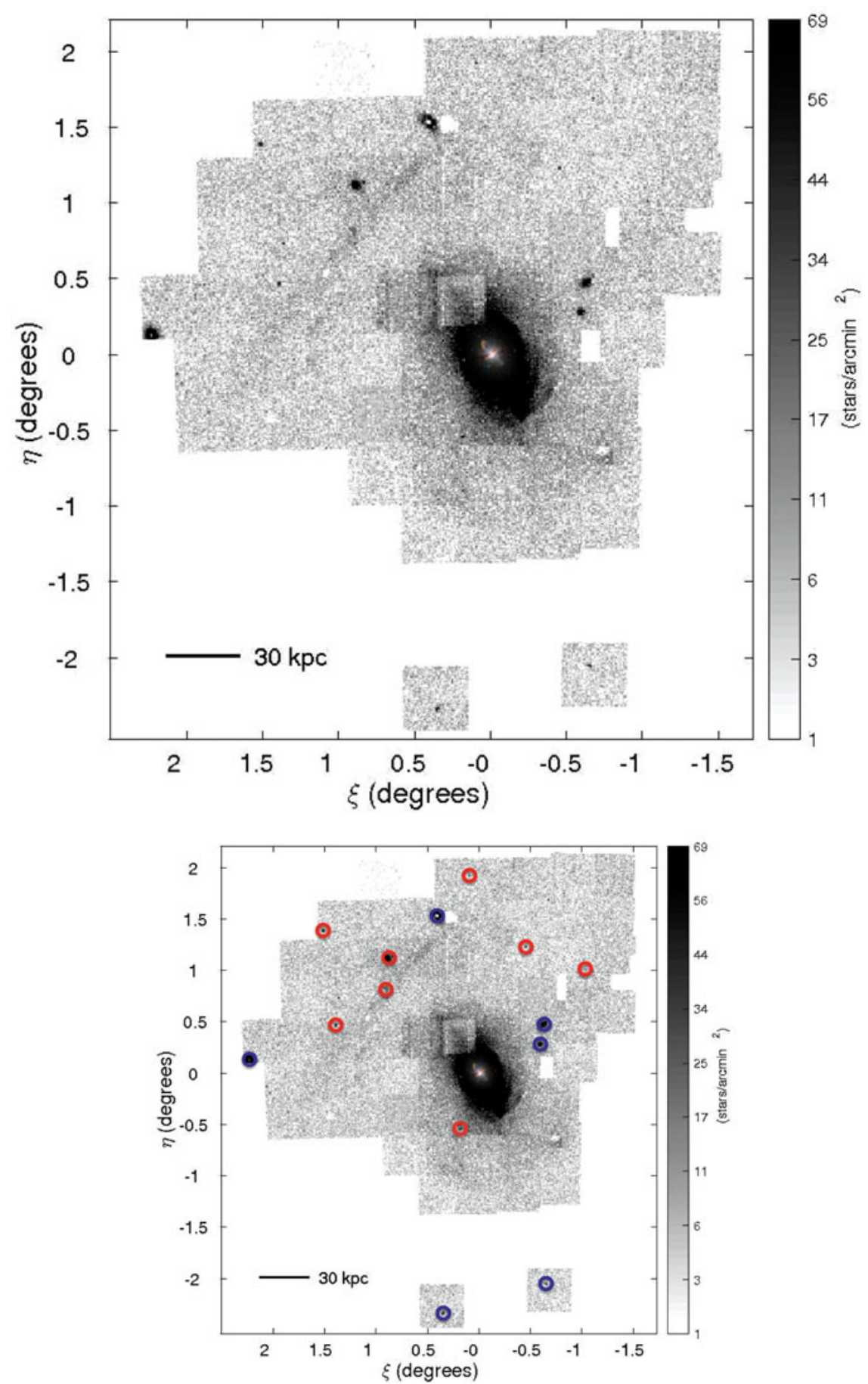

Figure 2. Stellar density map of metal-poor RGB stars (selected from the box in Fig. 1) for the halo of Cen A surveyed by PISCeS to date. Standard coordinates are centered on Cen A (N is up and $\mathrm{E}$ is left), and the central regions of the galaxy are replaced by a color image (credit: http://www.eso.org/public/images/eso0903a/); the star-count map in this region suffers from incompleteness due to high stellar crowding. The density scale as well as the physical scale are shown. In the lower panel, we indicate the position of the previously known (blue circles) and newly discovered satellites (red circles). 
the two bottom $(\mathrm{S})$ pointings. In the same map, we circle in red the nine robust new Cen $\mathrm{A}$ satellites, i.e. those that are firmly detected as RGB overdensities (we do not plot the four remaining candidates that are only seen as surface brightness enhancements). These newly discovered satellites are fainter than the known ones in terms of both absolute magnitude and central surface brightness, see e.g. CenA-MM-Dw1 and CenA-MM-Dw2 at $(0.9,1.1)$ (Crnojević et al. 2014), or even fainter candidates at $(1.5,1.4)$ (CenA-MMDw8), (-0.5, 1.2) (CenA-MM-Dw4), $(0.2,-0.4)$ (CenA-MM-Dw7) and $(0.9,0.8)$ (CenAMM-Dw3). The latter is the remnant of a galaxy caught in the midst of tidal disruption, with stunning tails extending in both directions over $1 \mathrm{deg}$ across the survey footprint (from E to NW). We stress that CenA-MM-Dw3 has extreme properties (very low central surface brightness coupled with large extent) with respect to LG satellites within the same luminosity range, and proves how sensitive our strategy is to such elusive objects. A similar potentially disrupting satellite, Scl-MM-Dw2, has also been uncovered in the vicinity of Sculptor (Toloba et al. 2016a).

\section{Conclusions and future work}

The PISCeS survey represents the next observational step in the future of near-field cosmology beyond the LG. PISCeS will charachterize in depth the resolved stellar halos of two $\sim \mathrm{MW}$-sized galaxies with different morphology and surrounding environments, and their newly discovered faint satellites and substructures. We have already secured followup imaging and spectroscopy of the newly discovered candidate satellites, streams and substructures with a multi-wavelength approach, namely optical with HST, near-infrared with Gemini/FLAMINGOS2, co-added spectroscopy (see also Toloba et al. 2016b) with both VLT/VIMOS and Keck/DEIMOS. Data have already started to be collected and will be in hand by the beginning of 2017. This strategy will allow us to derive: the profile, shape and extent of the targets' smooth halos and possible gradients in their resolved populations; the orbital properties of the uncovered streams which will constrain the host halo's mass; the relative mass contribution from in situ versus accreted stellar components; the faint end of the satellite luminosity function down to $M_{V} \sim-8.0$, as well as the star formation histories of the individual satellites discovered through our survey. Our results will be compared to extant wide- and narrow-field surveys of M31 (PAndAS; SPLASH, Gilbert et al. 2012) and of other nearby galaxies (e.g., M81, Chiboucas et al. 2013, Okamoto et al. 2015; GHOSTS, Monachesi et al. 2016). This will ultimately advance the observational census of galaxy outer halos and of their inhabitants, thus providing crucial constraints for current and future theoretical predictions of galaxy evolution.

\section{References}

Atkinson, A. M., Abraham, R. G., \& Ferguson, A. M. N. 2013, ApJ, 765, 28

Belokurov, V., Zucker, D. B., Evans, N. W., et al. 2006, ApJL, 647, L111

Boylan-Kolchin, M., Bullock, J. S., \& Kaplinghat, M. 2012, MNRAS, 422, 1203

Chiboucas, K., Jacobs, B. A., Tully, R. B., \& Karachentsev, I. D. 2013, AJ, 146, 126

Crnojević, D., Sand, D. J., Caldwell, N., et al. 2014, ApJL, 795, L35

Crnojević, D., et al. 2016, ApJ in press, ArXiv e-prints, arXiv:1512.05366

Dotter, A., Chaboyer, B., Jevremović, D., et al. 2008, ApJS, 178, 89

Duc, P.-A., Cuillandre, J.-C., Karabal, E., et al. 2015, MNRAS, 446, 120

Gilbert, K. M., Guhathakurta, P., Beaton, R. L., et al. 2012, ApJ, 760, 76

Ibata, R., Irwin, M., Lewis, G. F., \& Stolte, A. 2001, ApJL, 547, L133

Karachentsev, I. D. 2005, AJ, 129, 178

Martínez-Delgado, D., Gabany, R. J., Crawford, K., et al. 2010, AJ, 140, 962 
McConnachie, A. W., Irwin, M. J., Ibata, R. A., et al. 2009, Nature, 461, 66

McLeod, B., Geary, J., Conroy, M., et al. 2015, PASP, 127, 366

Merritt, A., van Dokkum, P., \& Abraham, R. 2014, ApJL, 787, L37

Moore, B., Ghigna, S., Governato, F., et al. 1999, ApJL, 524, L19

Monachesi, A., Bell, E. F., Radburn-Smith, D. J., et al. 2016, MNRAS, 457, 1419

Okamoto, S., Arimoto, N., Ferguson, A. M. N., et al. 2015, ApJL, 809, L1

Sand, D. J., Crnojević, D., Strader, J., et al. 2014, ApJL, 793, L7

Stetson, P. B. 1987, PASP, 99, 191

Stetson, P. B. 1994, PASP, 106, 250

Tal, T., van Dokkum, P. G., Nelan, J., \& Bezanson, R. 2009, AJ, 138, 1417

Toloba, E., et al. 2016b, submitted

Toloba, E., Sand, D. J., Spekkens, K., et al. 2016a, ApJ, 816, L5

Woodley, K. A., Harris, W. E., Beasley, M. A., et al. 2007, AJ, 134, 494 